\title{
Por una sociología de la estadística sobre la CIENCIA Y LA INNOVACIÓN*
}

\author{
BENOÎT GodiN**
}

\section{Resumen}

Las estadísticas son generalmente calificadas de objetivas, es decir, como si fueran reportes de hechos. Por esta razón también son presentadas como un instrumento esencial para la política pública. Éstas permiten esclarecer las decisiones políticas, objetivándolas y despersonalizándolas

La sociología de la ciencia tiene en cuestión esta aseveración desde hace largo tiempo, por considerar que las estadísticas son socialmente construidas. Por tal razón, se entiende que éstas llevan implícitas ciertas elecciones y ciertas decisiones que nada tienen que ver con la ciencia matemática, asunto que matiza los resultados obtenidos.

* El artículo original fue publicado en 2004, en el marco del "Project on the History and Sociology of S\&T Statistics" (Proyecto sobre la Historia y Sociología de las Estadísticas de Ciencia y Tecnología), y está disponible en: www.revue-lebanquet.com

** Profesor del INRS (Montreal); Profesor asociado de la Universidad Adolfo Ibañez (Santiago); Profesor invitado del MSH-CNRS (Paris); visitante asociado del Imperial College (Londres). Es Doctor en Estudios de Ciencia (Science studies), SPRU, Universidad de Sussex, Inglaterra, 1994 ; con Maestría en Ciencia Política (Science politique), Universidad Laval, 1984. Es fundador del Consorcio canadiense de indicadores de ciencia e innovación (Consortium canadien sur les indicateurs de science et d'innovation).

benoit.godin@ucs.inrs.ca 


\section{Résumé}

Les statistiques sont généralement qualifiées d'objectives, c'est-à-dire comme rapportant des faits. C'est pour cette raison qu'elles sont aussi présentées comme un instrument essentiel pour la politique publique. Elles permettraient d'éclairer les choix politiques en informant et d'objectiver les décisions en dépersonnalisant.

La sociologie de la science a depuis longtemps remis en question cette assertion. Les statistiques sont socialement construites. Par là, on entend qu'elles impliquent des choix qui reposent sur diverses considérations qui n'on rien à voir avec la science mathématique, choix qui colorent les résultats obtenus

\section{Abstract}

Statistics are generally classified as being 'objective', i.e. as if they were facts. Because of this, they are also referred to as essential instruments for public policies. This in turn allows the clarification of political decisions, making them more objective and less personalized.

The sociology of science has questioned this assertion for a long time, because it considers that statistics are socially constructed. Due to this, it is understood that they have some options and some decisions which are implicit and have nothing to do with mathematical sciences. A matter that qualifies the obtained results. 


\section{Presentación $* *+$}

Se decidió traducir este artículo para motivar una reflexión en torno a la forma como se utilizan las estadísticas relacionadas con la ciencia y la innovación. Es importante que los dirigentes gubernamentales, académicos y empresarios entiendan su mensaje central: Las estadísticas son constructos sociales y no simples reportes de hechos.

Cabe aclarar que no se pretende restarle importancia o credibilidad a dichas estadísticas, sino más bien resaltar la importancia de cualificar cada vez más la forma como nuestro país construye sus estadísticas, así como las correspondientes interpretaciones. Nuestros dirigentes deben comprender que, además de construir indicadores que permitan la comparabilidad a nivel internacional, es necesario construir socialmente otros indicadores orientados a entender los fenómenos socio-económicos desde una perspectiva propia, de tal forma que las estadísticas alimenten, con detalles más finos y análisis más profundos, las decisiones políticas en materia de ciencia, tecnología e innovación.

Se agradece al autor, Señor Benoît Godin, y al editor, Señor Nicolas Tenzer, por su autorización para traducir este interesante artículo.

\section{Por una sociología de la estadística sobre la ciencia y la INNOVACIÓN}

Las estadísticas son generalmente calificadas de objetivas, es decir, como si fueran reportes de hechos. Por esta razón también son presentadas como un instrumento esencial para la política pública.

*** La traducción del artículo es del profesor Fernelly Ruiz Gómez, Director de la Escuela de Egresados del Instituto Tecnológico Metropolitano, candidato a Doctor en Estudios sobre ciencia y tecnología, y Gestión de la innovación tecnológica. Ingeniero Electricista y Especialista en Telemática. Profesor de la cátedra de Ciencia, Tecnología y Sociedad del ITM. 
Estas permiten esclarecer las decisiones políticas, objetivándolas y despersonalizándolas ${ }^{1}$.

La sociología de la ciencia tiene en cuestión esta aseveración desde hace largo tiempo, por considerar que las estadísticas son socialmente construidas. Por tal razón, se entiende que estas llevan implícitas ciertas elecciones y ciertas decisiones que nada tienen que ver con la ciencia matemática, asunto que matiza los resultados obtenidos ${ }^{2}$.

Estas elecciones y decisiones se clasifican esencialmente en cuatro categorías: En primer lugar, 1) el fenómeno a medir (el qué), y 2) los instrumentos de medida (el cómo) orientados por esas decisiones que tratan de garantizar respectivamente su pertinencia y viabilidad. Y a su vez, estas dependen de: 3) los motivos detrás de la medición (el por qué), y 4) de quienes realizan la medición (quién). En resumen, la estadística está en función de una agenda a menudo más implícita que explícita.

Este artículo se dirige a la estadística oficial (gubernamental) sobre la ciencia y la innovación, una estadística que cuenta hoy día con más de 70 años. En efecto, durante los años 30, en el mundo anglosajón (Estados Unidos, Gran Bretaña, Canadá) ${ }^{3}$, fue donde las primeras estadísticas sobre la I+D (investigación y desarrollo) hicieron su aparición ${ }^{4}$. Su desarrollo subsiguiente y difusión dentro del conjunto de países occidentales, incluyendo a Francia, se debió en buena medida a que la OCDE estandariza un conjunto de opciones metodológicas en el seno de un manual conocido como el Manual de Frascati. [1]

1 Porter, T.M., Trust in Numbers: The Pursuit of Objectivity in Science and Public Life, Princeton: Princeton University Press, 1995.

2 Best, J., Damned Lies and Statistics: Untangling Numbers From the Media, Politicians and Activists, Berkeley: University of California Press, 2001.

3 Puesto aparte el mundo comunista (Mis à part le monde communiste).

4 Godin, B., The Number Makers: Fifty Years of Official Statistics on Science and Technology, Minerva, 40 (4) 2002: 375-397. 
Con respecto a la estadística oficial, dos tipos de agenda parecen haber gobernado respectivamente los ministerios y los organismos estadísticos durante el curso de dicho periodo. El desarrollo de actividades científicas y de innovación, además del apoyo a éstas, constituyeron el primer motivo de los gobiernos que inicialmente se interesaron por este tipo de mediciones. Es interesante contrastar los fines por los cuales las estadísticas de I+D son utilizadas especialmente por quienes las producen, fines que son relativos a los de otras estadísticas. La estadística económica y social tiende en general a sugerir el crecimiento de un problema: el desempleo es demasiado elevado, la inflación sube rápidamente, la pobreza es alta, etc., por lo tanto los gobiernos deben actuar. Contradictoriamente, en materia de ciencia e innovación, se asevera que las acciones no son suficientes: la I+D es insuficiente, no se dispone de una mano de obra suficientemente cualificada, etc. Es entonces, cuando los grandes números sirven para justificar la acción gubernamental en los dominios social y económico, ellos son, de manera casi universal, los que regulan la inversión en materia se ciencia e innovación.

La agenda de los organismos estadísticos oficiales es diferente y menos visible - pero no menos presente. En efecto, los organismos estadísticos no están para corregir sino para medir. En esta tarea involucran al menos tres "intereses". En primer lugar, una orientación (o ideología) relativamente economicista influencia la medición oficial de la ciencia y la innovación. No solamente se miden las actividades de acuerdo con las fuentes monetarias invertidas en ellas, sino que los principales indicadores, que muestran los resultados obtenidos, se concentran en los aspectos económicos de ciencia e innovación. Luego, la estadística es orientada dependiendo de las necesidades en política pública, más que por las tendencias del conocimiento: ella busca ser útil y responder a la estrategia del estado. En fin, los instrumentos utilizados para medir están impregnados de elecciones conceptuales y metodológicas que no pasan inocuamente sobre nuestra comprensión de la ciencia y la innovación. 
El presente artículo aporta una mirada crítica sobre la estadística relativa a la ciencia y a la innovación en un intento por responder a las cuatro siguientes cuestiones ${ }^{5}$ :

- Quién construye la estadística

- Porqué

- Qué se mide

- Cómo

\section{UN MONOPOLO DEL ESTADO}

Quien hoy desea hablar en términos cuantitativos de la ciencia y de la innovación no tiene otra opción que informarse sobre las estadísticas oficiales, con mayor razón si se quiere hablar de ciencia a nivel internacional. Este es un rasgo fundamental de la estadística sobre la ciencia y la innovación, las mediciones son realizadas por las oficinas gubernamentales y sus organismos estadísticos, más que por las universidades. En efecto, estas últimas son la fuente de desarrollo de varios conceptos y ejercicios preliminares de medición, al mismo tiempo que son consultadas por los ministerios y organismos estadísticos, pero los gobiernos disponen hoy de un monopolio sobre la producción de estadísticas sobre ciencia e innovación, monopolio que se refleja por otra parte en el hecho que las universidades se colman de estadísticas oficiales para alimentar sus trabajos. Este monopolio se explica esencialmente por el hecho de que los gobiernos son los únicos que disponen de recursos financieros suficientes para realizar las encuestas, sobre todo la repetición de encuestas que permitan disponer de series cronológicas a fin de seguir las tendencias en materia de ciencia e innovación.

Los recursos financieros, pero también los instrumentos de medición utilizados, explican dicho monopolio del estado. En efecto,

Godin, B., Measurement and Statistics in History of Science and Technology - 19302000, London: Routledge, sous presse. 
solo la encuesta por cuestionario es el instrumento legítimo de medición para los gobiernos. Se trata, por ejemplo, de cuestionarios dirigidos a empresas, para recoger información sobre los gastos y el personal activo en $\mathrm{I}+\mathrm{D}$. En general, toda estadística que no provenga de tales encuestas es desacreditada por los organismos estadísticos nacionales. Esto incluye la medida de la producción de conocimiento gracias a las denominadas publicaciones científicas (también llamada bibliometría) o la medida de la invención realizada a partir de las denominadas patentes. Los argumentos utilizados para rechazar estas herramientas se refieren a que las estadísticas producidas con la ayuda de sus datos no son confiables ni estandarizadas. En el fondo es debido a que esa información y los bancos de datos asociados a ella provienen de una fuente externa al organismo estadístico nacional, fuente que él no controla: las universidades en el caso de las estadísticas sobre las publicaciones, las oficinas de propiedad intelectual (ipor lo tanto públicasi) en el caso de las patentes.

Este control del instrumento (la encuesta por cuestionario) y de las fuentes estadísticas oficiales es fortalecido y cristalizado en un manual de estandarización que fue definido y ratificado por los países de la $O C D E$ : el Manual de Frascati ${ }^{6}$. El manual define los conceptos de base destinados a medir la I+D por encuesta y sugiere las reglas relativas a variables (o preguntas) a desarrollar, a fin de asegurar la compatibilidad de los datos entre países. Aparecido en 1963, el manual está hoy día en su sexta edición. [2]

El manual ha permitido que las estadísticas del estado obtengan una comprensión común de la $\mathrm{I}+\mathrm{D}$, aunque debe reconocerse que todavía es relativamente ambigua cuando llega el momento ser medida. Este manual cuenta con otra ventaja, el disponer de datos (relativamente) comparables entre países. Pero, a la vez, ha ayudado a los estados a monopolizar las mediciones de la ciencia y la innovación.

6 OECD, The Measurement of Scientific and Technical Activities: Proposed Standard Practice for Surveys of Research and Experimental Development, Paris, 2002. 
Para seguir profundizando en esta reflexión, y saber que se encuentra en la base de la medición de la ciencia y la innovación, se podría mencionar un país donde su influencia en los últimos 50 años ha sido cada vez mayor: los Estados Unidos. En los años 30 fue allí donde surgieron las primeras medidas de la $\mathrm{I}+\mathrm{D}$ en el mundo occidental. Posteriormente, veinte años mas tarde (1951), la Nacional Science Foundation (NSF) designada por el gobierno inicialmente para financiar la investigación fundamental en Estados Unidos, fue designada además para impulsar la Oficina de presupuesto (Bureau of Budget $(B o B)$ ), con el ánimo de controlar los gastos, así como cuidar la medición de las actividades científicas y tecnológicas ${ }^{7}$. Luego, al término de una decena de años de encuestas anuales, la $N S F$ dispuso de una experiencia relativamente única y de reflexiones metodológicas que sirvieron de base para la redacción del Manual de Frascati. La publicación de la serie titulada Science Indicators en 1972, recogió diversos indicadores bajo un mismo cuerpo, otra iniciativa de la NSF que sería luego imitada por un cierto número de países y de organismos internacionales.

Hace falta determinar que, si bien la NSF estuvo orientada decididamente a la investigación básica así como a sus análisis estadísticos, son los economistas quienes influyeron considerablemente en el conjunto de elecciones y consideraciones (percepciones de las cosas), y quienes constituyeron, para su análisis, la tercera fuente determinante de metodologías actuales, después de las oficinas de estadísticas de los Estados Unidos. Las estadísticas actuales sobre la ciencia y la innovación, así como los análisis basados en ellas, son el reflejo de su participación activa en el trabajo de los organismos estadísticos. Cualquiera que se interese por la medida de resultados derivados de las actividades de ciencia e innovación se debe ajustar a las estadísticas sobre patentes, sobre el balance de

7 Godin, B., The Emergence of Science and Technology Indicators: Why Did Governments Supplement Statistics with Indicators?, Research Policy, 32 (4), April 2003:679-691. 
pagos tecnológicos o sobre el comercio de productos de tecnología de punta. Se dispone, igualmente, de una vasta gama de estudios que buscan relacionar estadísticamente la $\mathrm{I}+\mathrm{D}$ y la productividad. Pero cuando llega el momento de medir otras dimensiones (medio ambiente, bienestar, salud, cultura), la estadística oficial es muda. Este hecho sorprendente, obligó a un cambio de "paradigma" de la política científica en los años 80 .

\section{Fines y usos políticos de LAS estadísticas}

Se distinguen generalmente dos periodos en la historia de la política científica. Uno que va aproximadamente de los años 50 a los años 70, caracterizado por el interés de los gobernantes hacia el desarrollo de actividades científicas y tecnológicas por la vía de su financiamiento, es decir, la política al servicio de la ciencia (policy for science). Luego, el periodo que sigue, será afectado por las consideraciones sociales - en términos políticos (militar, espacial) y económicos (industrial) - y buscará, con un relativo éxito, orientar el desarrollo científico con fines precisos, en este caso, la ciencia al servicio de la política (science for policy) ${ }^{8}$. A ambos periodos corresponden discursos y utilización paralelos de la estadística.

En el curso el primer periodo, la estadística fue utilizada por sus propios productores con el fin de elevar la ciencia y la tecnología al rango de prioridad presupuestal para los gobiernos. La $N S F$, por ejemplo, fue una ardiente defensora de la necesidad de invertir en ciencia básica. Esta investigación tuvo, desde V. Bush, las virtudes esenciales para impulsar el progreso, y es la estadística la que permite superar la simple retórica y mostrar los resultados. Las cifras indicaban que la investigación básica era un motor para el desempeño de la investigación aplicada, que las penurias de los investigadores se encontraban allí y que los Estados Unidos estaban a punto de ser superados en esta materia por la Unión Soviética9 .

\footnotetext{
OECD (1971), Science, Growth, and Society: A New Perspective, Paris.

9 Godin, B., Measuring Science: Is There Basic Research Without Statistics?, Social Science Information, 42 (1) Mars 2003: 57-90.
} 
Lejos de ser una postura revolucionaria, el aprovechamiento de las estadísticas no tuvo más camino que ser reemplazada por otro tipo de utilización a partir de los años 80: El uso de estadísticas para construir aquello que se llamó los marcos conceptuales destinados a destacar las intervenciones de la política científica. En el centro de estos análisis se encontraron los imperativos del progreso económico. La mayor parte de los escritos recientes de la $O C D E$ relativos al tema, donde se interpela la política científica - Sistema Nacional de Innovación, economía del conocimiento, competitividad, globalización, nueva economía- buscaron incorporar, con muchas dificultades, la co-relación entre I+D (o tecnologías de la información) y productividad.

Este tipo de trabajos no es más que uno de los numerosos usos actuales de la estadística. Se pueden identificar tres grandes tipos de utilización de ésta: teórica, práctica y política/simbólica. La primera utilización, estadística, es aquella en que las fuentes universitarias, por ejemplo, están destinadas a la comprensión de fenómenos relativos a la ciencia y la innovación. Los marcos conceptuales de la $O C D E$ son igualmente fuentes de la misma utilización. Ellos proveyeron a los gobiernos una forma de pensar la ciencia y la política científica, combinando elementos empíricos y comparativos. Fue así como la estadística llegó a alimentar las reflexiones conceptuales ${ }^{10}$.

Una segunda utilización de la estadística, en las relaciones con la política pública, es de tipo práctico. La estadística sirvió directamente a la toma de decisiones. Fue así como los gobiernos europeos se fijaron el objetivo de atender un nivel de gastos en $\mathrm{I}+\mathrm{D}$ equivalentes al $3 \%$ del PIB, desde la fecha de la decisión hasta llegar al 2010, o cuando Canadá se trazó el objetivo de obtener el quinto rango dentro de los países de la $O C D E$ sobre el mismo indicador; fue entonces la estadística quien dictaminó el objetivo ${ }^{11}$.

10 Godin, B., The Knowledge-Based Economy: Conceptual Framework or Buzzword?, Science, Technology and Human Values, à paraître.

11 Sheehan, J., and A. Wycoff, Targeting R\&D: Economic and Policy Implications of Increasing R\&D Spending, STI Working Paper, 2003, DSTI/DOC (2003) 8. 
Sin embargo, este tipo de utilización es relativamente rara en la historia reciente de la estadística. En general, la estadística, al menos la estadística oficial, arriba tarde para esclarecer las decisiones políticas, sin contar que ella es a menudo solo un agregado. Además, la política científica no reposa sobre ninguna legislación coercitiva o reglamentación que amarre imperativamente el uso de las estadísticas, contrariamente a otros dominios tales como la delimitación de las fronteras electorales, las cuales reposan sobre las estadísticas relativas a la población. Dicho de otra forma, la estadística oficial sobre la ciencia y la innovación se debe conformar con jugar un rol de naturaleza contextual: ayudar a configurar una imagen marco de las tendencias de ciencia e innovación, imagen que se encuentra, por lo general, dentro del capítulo introductorio de los documentos de política.

Un tipo de utilización de las estadísticas, más ampliamente difundido en la historia de la política científica de los últimos cuarenta años, es el simbólico y/o político. Se trata en general de convencer sobre la necesidad de invertir más en actividades de ciencia e innovación invocando las estadísticas como argumento o de exhibir mejores desempeños de los que las cifras muestran realmente.

En su historia reciente, Canadá ha mostrado ser un ejemplo de elocuencia en el uso político de la estadística ${ }^{12}$. En efecto, Québec se pone el reto a comienzos de los años 80 de diferenciarse de manera importante con respecto a Ontario en cuanto sus inversiones federales en ciencia y tecnología. Ontario recibía cerca del $60 \%$ de los aportes del gobierno federal en comparación con apenas un $14 \%$ por parte de Québec. Bajo el impulso (probable) de su Ministerio encargado (el de Industria en Canadá), Estadística Canadá tuvo la idea de generar sus estadísticas tendiendo a reducir la diferencia entre las dos provincias. Retira de las estadísticas la parte de los gastos federales correspondientes a la región de la capital nacional $(R C N)$, región que se soporta en las dos provincias y donde son

12 Godin, B., La distribution des ressources fédérales et la construction d'un territoire: la Région de la Capitale Nationale ( $\mathrm{RCN})$, Revue canadienne de science politique, 33 (2), 2000: 333-358. 
concentradas - en su parte de Ontario - los laboratorios federales. El artificio estadístico tuvo el efecto de pasar la diferencia del gasto entre Ontario y Québec a 8\% solamente. Así pues, Québec logra, de golpe, disponer de una relación $\mathrm{I}+\mathrm{D} / \mathrm{PIB}$ superior al de Ontario, algo que jamás se había presentado en su historia.

$\mathrm{El}$ análisis de los escritos de la $O C D E$ permiten observar un caso de utilización de las estadísticas con fines simbólicos. Se refiere a una práctica generalizada de comparación entre los países utilizando las mismas estadísticas de la $O C D E$. Con base en todos los análisis estadísticos obtenidos entre 1960 y 1979 , la $O C D E$ clasifica los países en función del indicador principal tomado del Manual de Frascati: la DIRD (Gasto Interno Bruto en I+D) y la relación DIRD/PIB ${ }^{13}$. Necesariamente, tales clasificaciones tienen efectos de imitación en los países que buscan compararse con los mejores desempeños, así estos últimos se convierten en símbolos de excelencia y, por el mismo hecho, en la norma. Los países que logran excelencia son en general muy rápidos para fijar sus desempeños, por aquellas razones que identificamos como políticas y simbólicas; y los que están en último rango se sienten muy tentados a exigirles a sus actores nacionales ciertos desempeños ubicados de acuerdo con las estadísticas de los más avanzados, en relación con su nivel de atraso. Durante esta época, Europa anhelaba el desempeño de los americanos y rápidamente su patrón de comparación estadística llegó a ser el reporte de la relación DIRD/PIB de Estados Unidos ${ }^{14}$.

\section{CONUENCIONES, POCO DICEN LAS CONVENCIONES}

La medición de la ciencia y la innovación es muestra de algunas dificultades considerables que nada tienen que ver con la matemática detrás de ella (de hecho, los manuales de la $O C D E$

13 Godin, B., The Most Cherished Indicator: Gross Domestic Expenditures on R\&D, Science and Public Policy, sous presse.

14 Godin, B., Technological Gaps: An Important Episode in the Construction of S\&T Statistics, Technology in Society, 24, 2002: 387-413. 
no se conducen ni mediante fórmulas matemáticas ni mediante simbolismos). Los principales problemas surgen por la definición de los conceptos a medir. ¿Qué es la ciencia?, ¿qué es la investigación?, ¿qué es la innovación? Las respuestas a estas preguntas reposan sobre una realidad empírica, ciertamente, pero solamente en parte. Pues la comprensión que se tiene de esos conceptos reposa igualmente sobre la forma como se ha medido durante cuarenta años. Y cuando llega el momento de medir, esas elecciones son realizadas por quienes levantan las convenciones[3].

La medición oficial de la ciencia y la innovación es bastante particular. En primer lugar, la mayor parte de las mediciones actuales no consideran más que a las ciencias naturales, médicas y la Ingeniería. Las ciencias sociales y humanas son raramente consideradas en la estadística. Sin embargo, existe un debate bastante antiguo sobre si esas disciplinas constituyen realmente unas ciencias. La estadística oficial responde de manera afirmativa, al menos en sus convenciones, pero la realidad es que no las considera en sus encuestas.

La estadística oficial mide la ciencia con la ayuda del concepto de investigación. La investigación que se mide hoy día tiene por nombre, y acrónimo, "I+D". Es necesario suponer, sin embargo, que este tipo de estadísticas hablen también de otras cosas además de la investigación estrictamente entendida. El Desarrollo "D", una actividad realizada esencialmente por las empresas, es la que constituye la parte más importante de la $\mathrm{I}+\mathrm{D}$ (aproximadamente el $70 \%)$.

Aún más, solo un tipo específico de $\mathrm{I}+\mathrm{D}$ es objeto de las encuestas. Por ser considerada como un fin de las estadísticas, la $\mathrm{I}+\mathrm{D}$ debe ser institucionalizada, es decir que debe ser realizada dentro de los cuatro siguientes sectores: industria, universidad, laboratorio público, organismos sin ánimo de lucro (Organisme sans but lucratif $-O S L B-$-). Pero sobre todo, la $\mathrm{I}+\mathrm{D}$ será medida correctamente si está localizada en un lugar preciso llamado laboratorio. Una $\mathrm{I}+\mathrm{D}$ realizada de manera atomizada o de manera irregular (no sistemática) será mal recogida por el instrumento 
de medida, e igualmente prohibida de medir. Desde hace mucho tiempo, y aún hoy en día, la $\mathrm{I}+\mathrm{D}$ realizada en las PYMES, la $\mathrm{I}+\mathrm{D}$ de las ciencias sociales y la $\mathrm{I}+\mathrm{D}$ desarrollada en el seno de las empresas de servicios, no es contabilizada a causa de esas convenciones.

La innovación evidencia los problemas de medición más significativos. En efecto, existen dos maneras de definir la innovación. $\mathrm{O}$ bien, la innovación es el conjunto de actividades destinadas a poner nuevos productos, procesos o servicios sobre la marcha (se habla entonces de la innovación). O bien, ella es el resultado de esas actividades: un nuevo producto, un nuevo proceso, un nuevo servicio (se habla de una innovación). La estadística oficial escogió medir la innovación en tanto que actividad ${ }^{15}$. Las razones para esta decisión son múltiples, pero la lógica de los organismos estadísticos, que miden las actividades, es determinante - sobre todo cuando las fuentes para medir la innovación en tanto que resultados (output) no descansan en la encuesta por cuestionario.

La maleabilidad del concepto de innovación no se detiene ahí. Una empresa innovadora no es solamente una empresa que realiza actividades de innovación o que inventa nuevos productos o servicios. Es igualmente una empresa que adopta nuevas tecnologías simplemente para mejorar su desempeño y su productividad. Esta es una dimensión importante para que la empresa sea "tecnológicamente avanzada", lo que vuelve a poner en cuestión las estadísticas sobre la tecnología de punta que se desprenden las mediciones de la $\mathrm{I}+\mathrm{D}$, que no consideran el estado de los equipos de la empresa, es decir, la $\mathrm{I}+\mathrm{D}$ incorporada dentro de los bienes adquiridos por una empresa.

Definir los conceptos no es más que uno de los aspectos relativos al objeto que se desea medir. Después se debe clasificar dicho objeto según las dimensiones que dan sentido a las estadísticas. Aquí debe mencionarse que la $\mathrm{I}+\mathrm{D}$ es medida para cada sector institucional

15 OECD/Eurostat, Proposed Guidelines for Collecting and Interpreting Technological Innovation Data (Oslo Manual), Paris, 1997; Godin, B., The Rise of Innovation Surveys: Measuring a Fuzzy Concept, comunicación presentada en la conferencia internacional en honor de K. Pavitt "What We Know About Innovation", 13-15 Novembre 2003, SPRU, University of Sussez, Brighton (UK). 
definido, es decir, las empresas, los laboratorios públicos, las universidades y las entidades sin ánimo de lucro (OSLB). Las estadísticas generalmente se presentan y se analizan de acuerdo con esta clasificación. Cada sector es tomado por el Manual de Frascati de acuerdo con las clasificaciones existentes, especialmente las de la UNESCO. Las empresas se asocian a una clasificación de la I+D por sectores industriales (-ISIC International Standard Industrial Classification); los ministerios se clasifican según los objetivos socioeconómicos que involucran las intervenciones del estado, clasificación inspirada en los documentos presupuestales (-NABS-Nomenclature pour l'analyse et la comparaison des budgets et des programmes scientifiques); las universidades según una clasificación disciplinaria (de tipo-CLARDER-Classification et regroupement des domaines d'enseignement et de recherche).

Es más tarde que se percibe la dificultad de enlazar el papel del estado con las estadísticas así concebidas. Cada sector disponía de su propia clasificación, por tanto era imposible establecer una relación entre los esfuerzos gubernamentales dentro de un sector dado, por ejemplo, y su impacto bajo la industria correspondiente. Esto, en tanto que se encasillaban las clasificaciones de la $\mathrm{I}+\mathrm{D}$ dentro de moldes que no correspondían verdaderamente a la realidad: la investigación universitaria, por ejemplo, es cada vez más multidisciplinaria que disciplinaria; la investigación empresarial está orientada según los múltiples productos en el seno de una misma industria y que no se reducen a la categoría de una única industrial.

La clasificación de la I+D en básica o aplicada surge de la misma lógica, es decir, de someterla a las clasificaciones existentes. Esta dicotomía, que existe desde hace mucho tiempo, y que sirvió a los investigadores universitarios durante varias décadas, continua en la estadística a partir de la primera edición del Manual de Frascati ${ }^{16}$. Sin embargo, muy pronto, un buen número de países cesaron de recoger la información bajo esta dimensión de la investigación. En efecto, clasificar los proyectos de investigación

16 Godin, B., Measuring Science: Is There Basic Research Without Statistics?, op.cit. 
según estas categorías surge a menudo de la arbitrariedad. Diversas soluciones que fueron surgiendo en el curso de los últimos treinta años-algunas de las cuales añadieron una categoría de investigación que se denominó orientada o estratégica entre las dos, recomendada entre otras cosas dentro del Manual de Frascati - pero ninguna se acomodó realmente a las estadísticas del estado. [4]

En resumen, si el Estado se apropia de la medición de la ciencia y la innovación, tiene que dejar a otros el cuidado de definir la forma como presentará sus estadísticas. La estadística sobre ciencia e innovación reconduce las elecciones históricas que a menudo son percibidas como dicotomías de toda suerte. Si la medida de la ciencia y la innovación abunda en dicotomías, es porque la elección de medir tal objeto de tal manera $u$ otra conduce a ciertas exclusiones y, consecuentemente, a oposiciones: ciencias naturales versus ciencias sociales, $\mathrm{I}+\mathrm{D}$ versus actividades científicas denominadas conexas, innovación tecnológica versus innovación social, investigación básica versus investigación aplicada.

\section{Lo QUE NO SE DICE DE LA MEDICIÓN}

La medición de la ciencia y de la innovación ha seguido un desarrollo diferente dependiendo de la época, hasta que finalmente dispuso de sus propias herramientas. Las primeras estadísticas sacaron sus fuentes de datos existentes, tales como los directorios de laboratorios industriales. Se constituían entonces lo que se llamó las variables "Proxy" para medir los fenómenos. Por ejemplo, la innovación fue durante largo tiempo medida con la ayuda de la $\mathrm{I}+\mathrm{D}$, si bien la primera cubre un conjunto más amplio de actividades. Después, vino la encuesta específicamente dedicada: encuesta sobre I+D y después, en los años 90, encuesta sobre innovación. Finalmente, los estándares fueron desarrollándose a nivel internacional buscando armonizar las metodologías de colección de datos entre países. La última innovación a la fecha es la concepción de tablas de puntuación (scoreboard) que reúnen en un solo documento los indicadores extraídos de diversas fuentes 
y destinados a comparar los países bajo múltiples dimensiones de ciencia e innovación ${ }^{17}$.

Debe admitirse, que existió un sesgo a lo largo del desarrollo de la medición. Medir siempre, después de 60 años de trabajo estadístico, las entradas, o las fuentes involucradas en las actividades científicas y tecnológicas, pero muy poco las salidas y los impactos, es decir los resultados desprendidos de la ciencia y la innovación. En efecto son relativamente fáciles de contabilizar las cantidades invertidas en dichas actividades. Esta es la lógica del Estado-y de los economistas. En realidad los retos son otros - no insolubles- en cuanto a la medición de resultados que permanecen a veces intangibles, sin olvidar los que se manifiestan, a menudo, a más largo plazo.

No obstante, desde hace decenios, la estadística ha sido mejorada considerablemente. Sería erróneo pretender, por esta razón, que se midan perfectamente los fenómenos estudiados. Recientemente, por ejemplo, un hecho muy llamativo se manifestó en el análisis de unos resultados de encuestas. Se evidenció que las cifras obtenidas sobre las investigaciones en $\mathrm{I}+\mathrm{D}$ diferían más que otras, de manera muy apreciable, según provinieran de la encuesta sobre I+D o de la encuesta sobre innovación ${ }^{18}$. Un caso evidente donde el instrumento utilizado tiene un impacto directo sobre los resultados obtenidos.

17 Esos documentos son raramente producidos por los organismos estadísticos nacionales, mas bien por los organismos internacionales como la OCDE, o regionales.

18 OECD, Assess Whether There Are Changes Needed as a Result of the Comparison of R\&D Data Collected in R\&D and in Innovations Surveys, 2001, DSTI/EAS/STP/ NESTI (2001) 14/PART3; D. Francoz, Measuring $R \& D$ in $R \& D$ and Innovation Surveys: Analysis of Causes of Divergence in Nine OECD Countries, 2000, DSTI/ EAS/STP/NESTI (2000) 26; D. Francoz, Achieving Reliable Results From Innovation Surveys: Methodological Lessons Learned From Experience in OECD member countries, Comunicación presentada en la conferencia sobre "Innovation and Enterprise Creation: Statistics and Indicators", Sophia Antipolis, 23-24 November 2000. Ver también, para Italia y Alemania: G. Sirilli, Old and New Paradigms in the Measurement of $R \& D, 1999$, DSTI/EAS/STP/NESTI (99) 13; C. Grenzmann, Differences in the Results of the R\&D Survey and Innovation Survey: Remark on the State of the Inquiry, 2000, DSTI/EAS/STP/NESTI/RD (2000) 24. 
Las estadísticas sobre ciencia e innovación permanecen impregnadas de importantes límites metodológicos, dentro de los cuales el primero, como ya se dijo, se relaciona con los conceptos a medir. Este hecho es admitido por los estadísticos-quienes, sin embargo, no ven los límites de sus propias estadísticas cuando llega el momento de criticar las fuentes de aquellos datos provenientes de los organismos estadísticos nacionales. Así, un lector atento observará en las tablas estadísticas las notas metodológicas, con el fin de cualificar los datos: su calidad, su inclinación, etc.

Sin embargo, las notas metodológicas no siempre están bien detalladas. Ellas toman más la forma de notas estandarizadas o aparecen en documentos separados, que no favorecen una lectura crítica de los datos. En efecto, quienes las utilizan tienden en general a no considerarlas (tal es el caso de los documentos de política), y quienes las producen tienden a minimizarlas, argumentando que son solo las tendencias las que cuentan ${ }^{19}$. Es de esta manera que las estadísticas ganan en autonomía y legitimidad. Ellas circulan sin reserva ni precaución. Es entonces cuando se toman como la realidad y se olvida que una estadística es siempre socialmente construida.

\section{Conclusión}

Los gobiernos y sus organismos innovaron cuando, hace 70 años, se encontraron con la necesidad de medir la ciencia y la innovación. Una comunidad de investigadores universitarios, principalmente compuesta por economistas, desarrollaron formas de utilizar esas estadísticas, y luego se orientaron a producirlas (cada quien de manera diferente), sin olvidar su participación en los esfuerzos de los organismos estadísticos.

Los vínculos que se tejen entre la estadística y la política son múltiples. Van desde la comprensión de los fenómenos sobre los

19 B. Godin, Metadata: How Footnotes Make for Doubtful Numbers, Project on the History and Sociology of Science and Technology Statistics, Montreal, 2002: http://www.inrs-ucs.uquebec.ca/inc/godin/metadata.pdf 
cuales se va a intervenir (teórico) a la evaluación de las acciones gubernamentales (práctico), sin olvidar la promoción de los esfuerzos nacionales (simbólico). Si la estadística oficial juega un rol importante en este último punto, este no depende solamente de considerar estas otras dos funciones. El análisis cualitativo es omnipresente en el primer caso, y la estadística administrativa es determinada por la segunda.

Hace falta que la estadística oficial contribuya a la política pública, vía la cristalización de los conceptos que ella involucra. Es ella quien dio cuerpo a los recientes "buzzwords" que fundamentan la política científica: tecnología de punta, globalización, sociedad de la información, economía del conocimiento, nueva economía. Sin ella, los discursos sobre estas nociones tendrían, probablemente, mucha menos influencia.

\section{NOTAS DEL TRADUCTOR:}

[1] La OECD es la "Organisation for economic co-operation and development" (Organización para la cooperación y el desarrollo económico). Es un foro donde 30 gobiernos democráticos trabajan por dirigir los cambios económicos, sociales y ambientales de la globalización. En adelante se mencionará mediante su sigla en Francés y Español "OCDE".

[2] En 2005 fue publicada la última versión del Manual de Oslo: OECD (2005), Oslo Manual, Guidelines for collecting and interpreting innovation data, $3^{a}$ edición. Centre Francais d'explotation, Paris (Francia). Disponible en: http://www.oecd.org

[3] Así se interpreta, de acuerdo con el texto de este aparte, el subtítulo "Des conventions, rien que des conventions".

[4] La investigación "orientada" o "estratégica" que se ubica entre la investigación básica y la aplicada es aquella con la que pretenden encontrar nuevas aplicaciones al conocimiento científico que se produce, o nuevas necesidades de producción de conocimiento científico para ser aplicado luego. 
